\title{
Theoretical Design of Intelligent Campus Bus System -Using Wuhan University of Technology for Example
}

\author{
Huan Wang \\ Wuhan University of Technology \\ No.122, Luoshi Road \\ Wuhan, China \\ e-mail:wanghjoy@163.com \\ Xiao-Ru Zhao \\ Wuhan University of Technology \\ No.122, Luoshi Road \\ Wuhan, China \\ Hui-Ying Nong \\ Wuhan University of Technology \\ No.122, Luoshi Road \\ Wuhan, China
}

\author{
Hong Liu \\ Wuhan University of Technology \\ No.122, Luoshi Road \\ Wuhan, China \\ Jia-Yang Wang \\ Wuhan University of Technology \\ No.122, Luoshi Road \\ Wuhan, China
}

\begin{abstract}
This paper is to address the problem about the unreasonable scheduling of school buses, which is caused by the reason that the supply and demand information cannot be real-time communicated in campus traffic. The framework of intelligent school bus system is built by data integration, information interactive technology. Using GPS technology it gets school buses and passenger location information. Then it combines GIS spatial data with student information database to refine the internal functions. The whole system writes by Java to support it running on the Android platform. The system has interactive functions such as real-time queries, sends a travel request, confirm travel information at last. This system is a custom-built campus traffic system, makes school bus real-time scheduling better. It breakthrough sets integrity evaluation module in functionality, optimized the management.
\end{abstract} GIS.

Keywords-intelligent school buses;Android platform GPS;

\section{PREFACE}

Reasonable traffic system design and management have vital significance to ensure the safe and smooth traffic operation of urban complex and urban traffic system [1]. Campus intelligent traffic is a specific application category of ITS (Intelligent Traffic System), but it hasn't be fully used on campus of China. Thus, most universities have such problems that the area of their campus is too big for school buses to play their part. Meanwhile, mobility and huge number of students, in addition of concentrated demand time and hysteretic information cause the unreasonable scheduling and further lead inconvenience of taking classes and more pollutants of vehicle scheduling.
To solve the above problems, this paper narrates the process of designing an intelligent school bus management system and realizing the optimization of school bus. The paper takes Wuhan University of Technology as example. Based on Android mobile client, we use GPS, GIS and Internet technology to realize visualization of geographic coordinates. At the same time, a custom-built campus traffic system is designed. It combines intelligent school bus system and information of teachers and students by using java language and algorithm.

\section{SYSTEM REQUIREMENT ANALYSIS}

Demand determination is the less technology-required stage of system development, but if this stage hasn't fully completed, the result will be worse than not completed other stages [2]. Diversification of people's requirement and improvements of technology are two main motive power in the process of ITS development, while it is requirement that has pushed ITS forward fundamentally. So, to design it better, it is very necessary to analyze the relevant requirement.

\section{A. Functions Required}

There must be a clear objective, in this time; we will analyses by questionnaires and open interviews. The number of student from different campus is even and the whole number is huge in our research, following analyses are representative. There is $92.31 \%$ of the student shear the point that an intelligent school bus system is needed. In the survey, we analyze students' demand for functions by the two following figures.

Figure 1 and Figure 2 are pie charts of students' disadvantages and functions in expect. From the figure, we 
can know that most students are not satisfied with some aspects, such as large randomness, little number of the school buses, inaccurate timetable and unable to take the school buses in the evening. To sum up, the problems are chaotic crowds, inadequate buses, and inaccurate timetable of the buses. The corresponding function which most students hope the application has is checking real-time information. The purpose of the intelligent school bus application is solve the matters about we students take the school buses. Therefore, the core function of the application is checking real-time information. The other functions in figure2 such as reserving the school buses, evaluating the school buses, pay for the fare, will also be realized. As noncore functions, we will simplify them. In addition, we also conducted a survey of integrity which shows $97.44 \%$ of the students are willing to follow the letter of credit on the App. It can reduce the trouble of scheduling for school bus drivers, and to achieve the optimization of school bus resources.

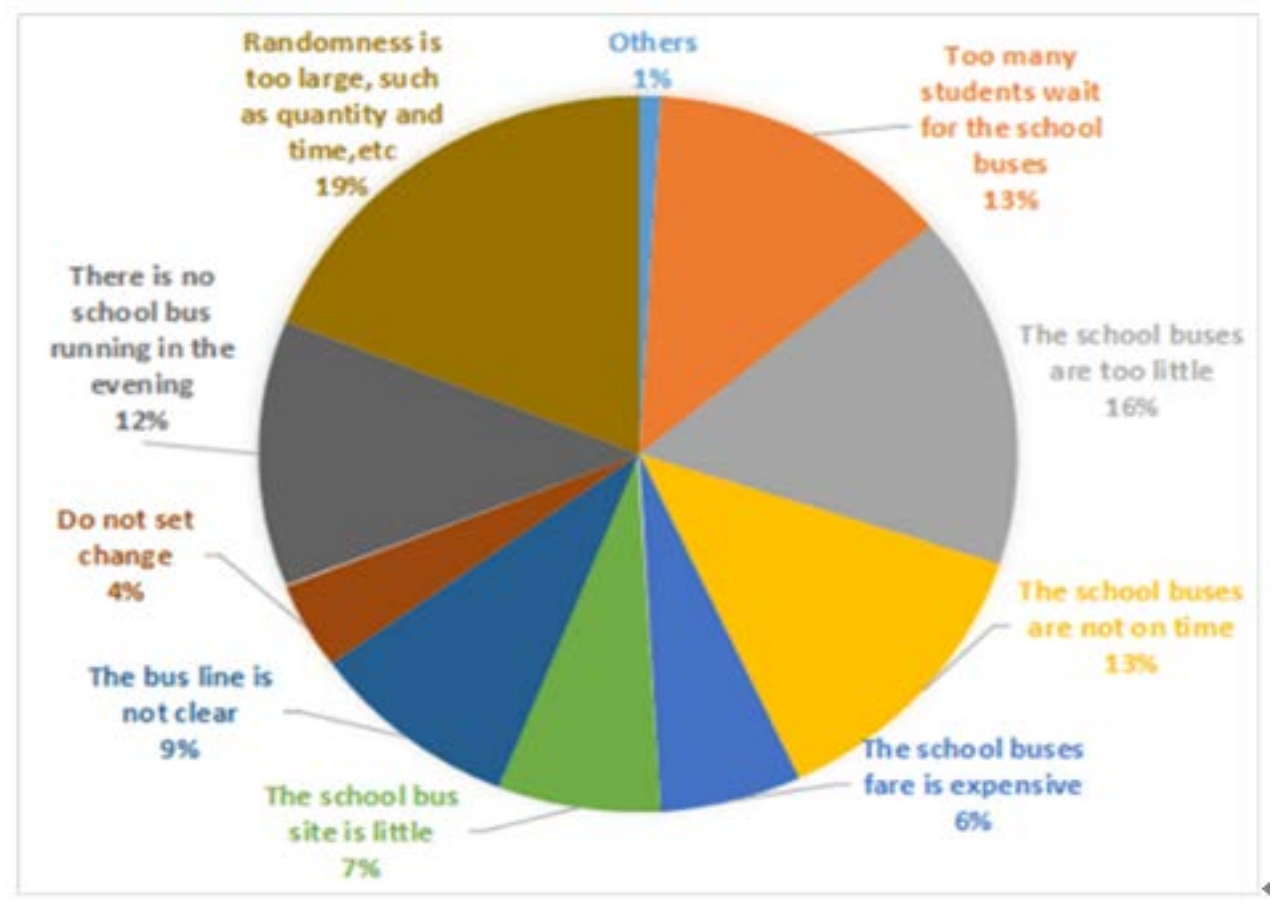

Figure 1. shortcomings

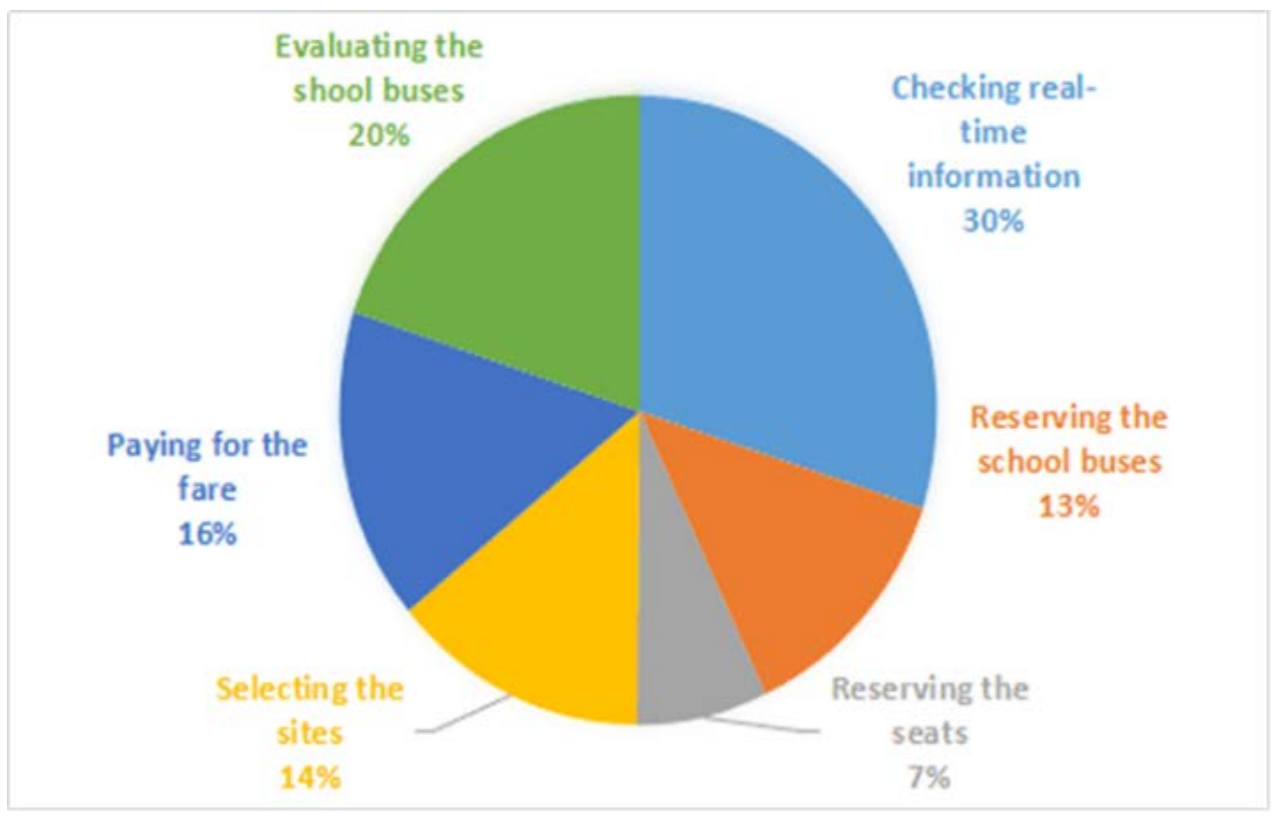

Figure 2. Function to be realized 


\section{B. Non-functional Requirements Analysis}

Technically, performing rational analysis on GPS data and school traffic information by using MATLAB, Genetic Algorithms Theories and other methods, having the ability to do programming based on JAVA and Android. Besides, the application we made should meet the following requirements: the reliability related to the ability of software to maintain its performance level in a certain period of time and condition; the easily use related to the effort a group of regulated or potential users have to take to use this application , and the estimation about it; the efficiency, efficiency means a list of attributions of the software's performance under a certain condition and the resource amount it consumes; the portability related to the software's ability to move from a certain environment to another.

In other ways, for example, the workability is required to be easy to use and have a friendly interface. As for security and secrecy, if the data, like the route of school bus can be tampered at will, there would be a great mess. To avoid this, the software needs to have the login cipher test function, and the cipher can be changed after. Finally, the maintainability requires our software has a full set of maintenance document, which would be convenient for software maintenance.

\section{Overall System Architecture Design}

Intelligent transportation system is an open complex giant system, which is composed of many subsystems closely related to different functions. It has the characteristics of multi subject, cross sector, cross domain, complexity, and system characteristics [3]. As for intelligent school bus system, its core is control, the same as the traditional ITS-integrate the control technology ,information technology and communication technology into the traffic filed, forming a completed control system. The difference is that the intelligent school bus system is targeted, so the whole frame is simple and applying easily. It meets the universities' requirements while it can be promote and reference.

\section{A. The Hierarchical Structure of System}

The hierarchical structure of intelligent school bus system shown in Figure 3, both the vehicle terminal and the client end are in the same level. Both of them are communicating with the dispatching center through the communication network. The campus card system is mainly used for the user to confirm the landing and car confirmation. There is data integration and one-way calls between the external subsystem and the campus card system. The client mainly implements certain functions which release passenger demand and receive feedback. The dispatch center is responsible for route planning and decision distribution according to the user's demand information, at the same time, archiving the scheduling information. The vehicle mounted terminal is used for receiving the allocation decision of the dispatch center and to execute, the real-time positioning data is sent to the client at the same time. After the arrival of the school bus, the vehicle mounted terminal is doing the integrity assessment for the user according to the situation of their campus card. Campus card system, which is complete and encapsulated, mainly plays the function of student information confirmation and bus payment in the intelligent school bus system. The system which is mainly based on GIS, GPS and mobile communication networks that these are relatively mature technology. It improves the feasibility at the same time reduce the complexity and redundancy of the system, saving the system construction costs.

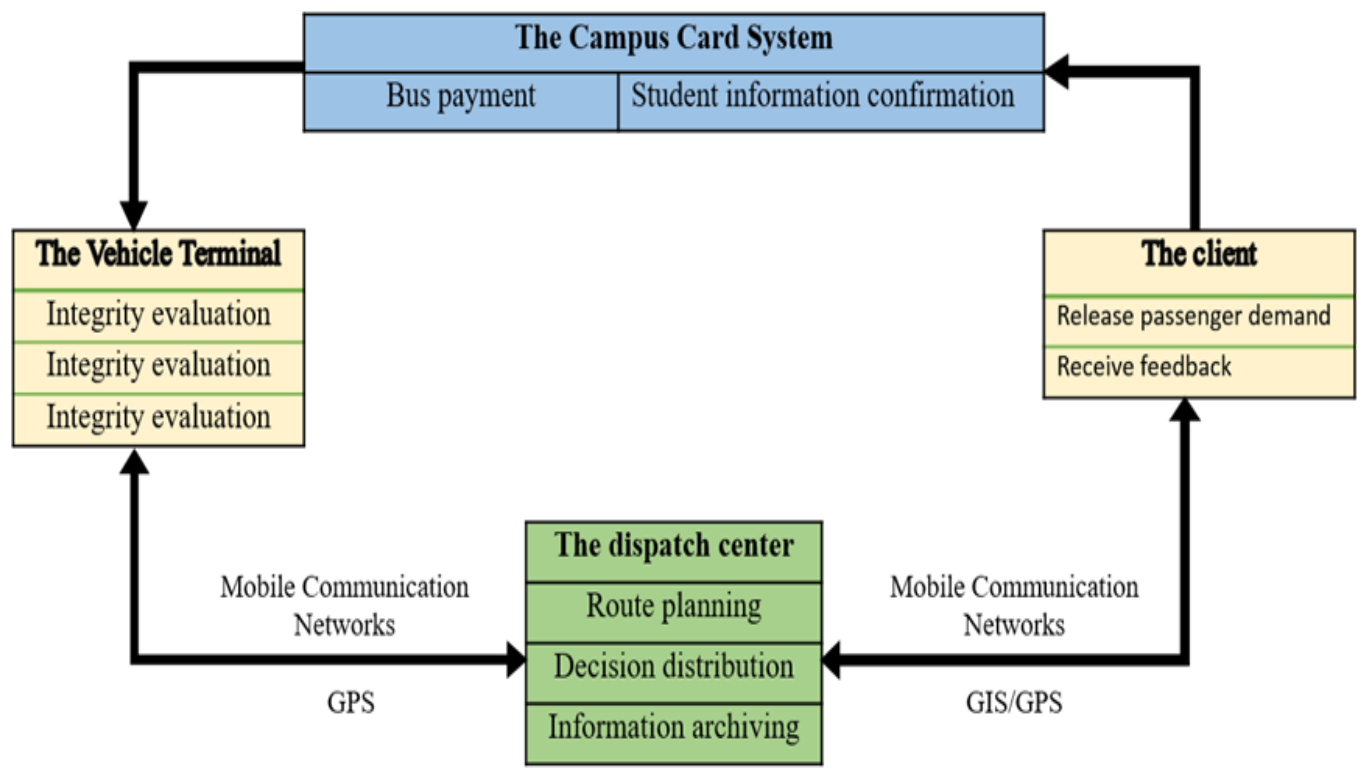

Figure 3. the hierarchical structure of system 


\section{B. The information flow of system}

The information flow of intelligent school bus system is shown in Figure 4. The terminal of school bus and the client carry on information transmission with traffic dispatch center to realize the school bus-the user's real-time traffic information interaction. At the same time, the campus card which is as a user database system provides login data to support payment confirmation and integrity evaluation. As shown in Figure 4, the real-time location data of school bus and passenger demand belong to upstream information. The upstream information convey to the dispatching. The decision information and distance information through the dispatch center processing belong to downlink information.

In the process of data flow and processing, the key points are real-time interaction and data integration.

\section{THE FUNCTION OF THE SYSTEM}

\section{A. Online Inquiry Module}

The intelligent campus traffic system based on Android platform is a comprehensive data querying system showing map layer together with buses layer which combines map information with bus lines information. It makes the scheduling more convenient, including less number of bus.
Therefore it is extremely necessary for the user to query the number of bus in time and realize the real-time scheduling of school bus.

Online query is one of the most important functional modules of the system, the school bus online query consists of the client and vehicle terminal. According to passenger demand, the main interface of client can choose inquiry modes, which is station enquiry and bus line query, the default setting for the station query [4].

- When choosing the site enquiry, the user enters into the starting and destination according to their requirements, queries the exact information from the database. The system parses feedback data of the server, display detailed information about road line and the line of site lists. It also shows the nearest school bus position from the destination site and automatically refresh [4] to display the real-time information.

- When choosing the tine enquiry, the user enters into the information of starting station and terminal. Then the system displays the information which is all sites on the line in accordance with the direction for the first time after the database query.

The program flow diagram:

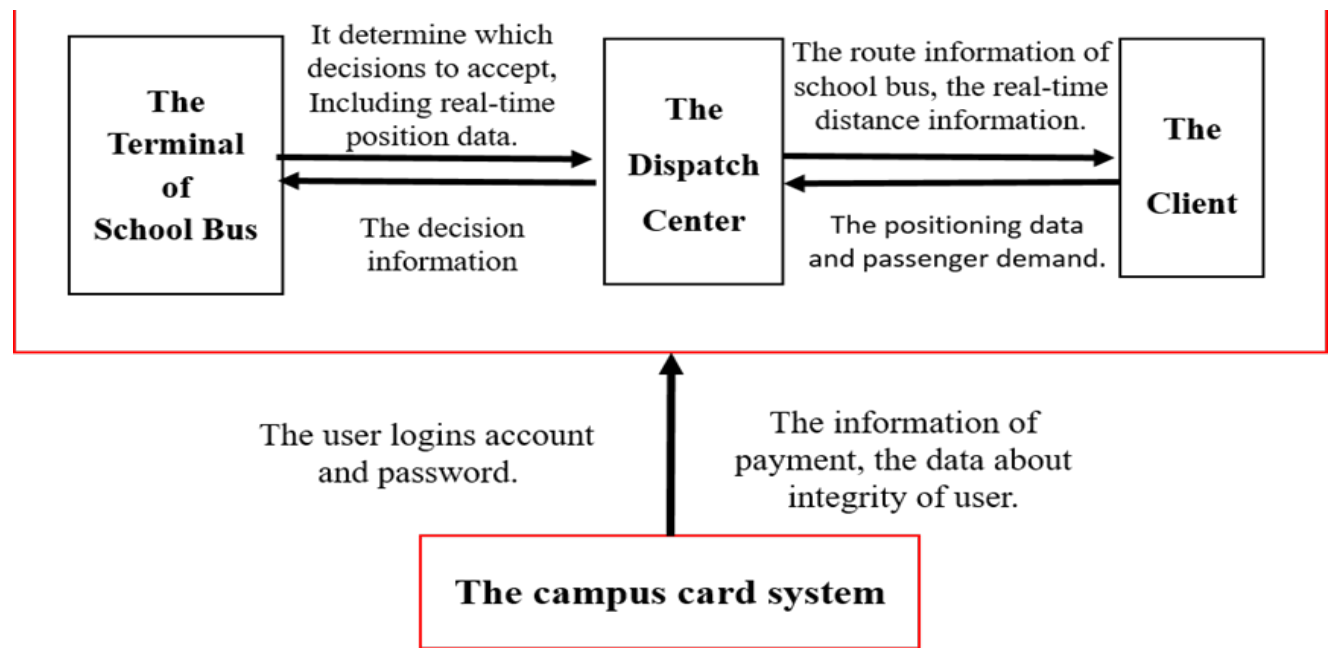

Figure 4. the information flow of system 


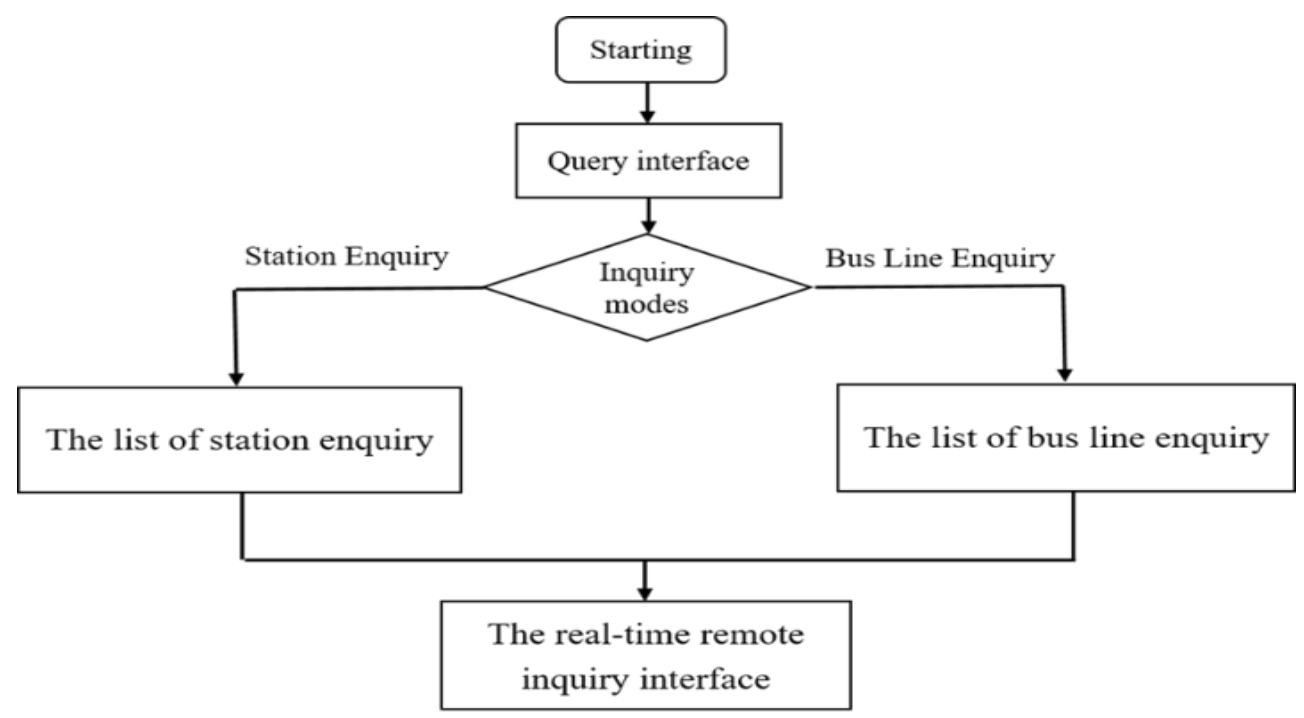

Figure 5. Online query flow chart

\section{B. Data Integration Module}

Due to the present information system construction which has the characteristics of periodic and distributed, there will be the existence of "information island" phenomenon. It will cause a large number of redundant data. The central task of data integration is that integration the distributed heterogeneous data sources which are interrelated, enabling users to access these data sources in a transparent manner. Integration refers to maintain the data consistency on the whole data source, increasing the efficiency of sharing information; transparent way refers to that users need not care about how to realize the access of heterogeneous data sources data, only care about in which way to access which data [5].

In order to achieve all kinds of information that from system's real-time circulation, call, processing to make users have more convenient using experience, this article integrated multiple system data such as the basic information data between teachers and students, campus card consumption data, GPS data, the campus traffic data.

\section{Aims of Data Integration}

Data integration's aim is to provide a unified access to the set of autonomous and heterogeneous data sources. Intelligent school bus system links different data sources together through data integration system, to improve the accuracy of the system. In this paper designed the intelligent school bus system, that with the aid of data integration to achieve the three main purpose:

- Integration of three data that are information data between teachers and students (school bus users), campus card consumption data and client location data to realize the integrity evaluation function of the system.

- Integration of the campus traffic route data and GPS information which based on digital map, realizes the vehicle terminal and user location visualization.
- Integration of the user's demand information data, the vehicle terminal system to locate user's location, and according to the number and destination which are displayed by it, it will be convenient for route planning.

\section{Realization of Data Integration}

Intelligent school bus system need to interact with every information system. The interface mode mainly is the Web Service, which uses the Web Service communication mode to exchange the data between platform and system then display information, using SOAP protocol to interact. Interactions of the underlying communication protocol are the HTTP protocol, data transmission format for XM [6].

When system begins to integration data, it need to integration heterogeneous data in a unified data format, which facilitate calling. This paper adopted the XML data format. XML is a commonly used data format, provides a unified way to describe and exchange structured data which is independent of the application program, it is a crossplatform, depends on the content of technology in the Internet environment, it's also the effective tool when dealing with distributed structure information today. XML data document is the carrier of passing data, the basic content including file header information, master data, and additional data part. System in the region of the header files stores information such as the school bus time of beginning and ending and basic line. Storing the passenger information, such as personal information, geographic coordinates, and ride requirements in the client identity.

When intelligent school bus system and data platform exchange data, it also needs to process with the data such as error correction code, code standardization, field split, format conversion, in order to form the standardized data.

\section{E. Real-time Interaction Module}

Real-time interactive module was one of the most important functions in intelligent school bus system, mainly 
used to solve the problem that information of passengers and school bus cannot flow and communicate. Each user can interact by level of information query and uploading, returns the user feedback through interactive level. Campus card system is used to store users ' personal information and provide client login information. Clients include user login module, GPS module, information transmission and data receiver module on the school bus. Required information interaction includes ride information data and feedback received and credibility assessment.

There reserves a vehicle for information exchange between the passengers and school bus (Figure 6). User-end passengers login in the app by their campus card account and password and then through the search function in the mobile phone to display location and number of school bus. Passengers selected station and timely confirmation of the school bus. Then the dispatch center for data clients receive after booking success with school bus routes, departure time and distance information. School bus vehicle-side position is related to school buses that pass through network location, number and other information. Accept passengers from the dispatch center data, departure time and route information and perform. Dispatch Center received the demand information from the client over the network, and in-vehicle location and quantity information from end, processing module of automatic discovery of storage in the system is running the best route data, statistics for each site number of passenger information, plan routes, and make a decision.

Among them, the best route is to use GIS designing and planning of school bus routes. First managed in ArcGIS school bus parked in each campus, student travel sites, traffic routes and other geographical data [7]. Using ArcView software and accompanying analysis of network expansion module (Network Analyst) for analysis. The network analysis module is an extension of path analysis based on ArcView development modules, it can help manage the complex network data collection provides a solution for network-based spatial analysis. [8]

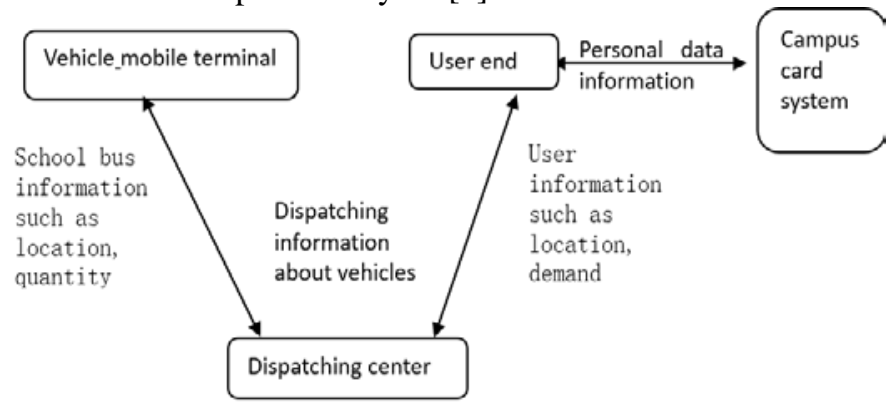

Figure 6. Real-time interactive module

\section{F. Credibility assessment}

Credibility testing is mainly to avoid the waste of resources caused by malicious call a cab. Its realization is given a certain amount of credit points, if the user has sent travel demand but not take buses and fail to cancel the call, credit points are deduct. At last, the punishment is that score is too low to use this app for students. The flow chart in Figure 7.

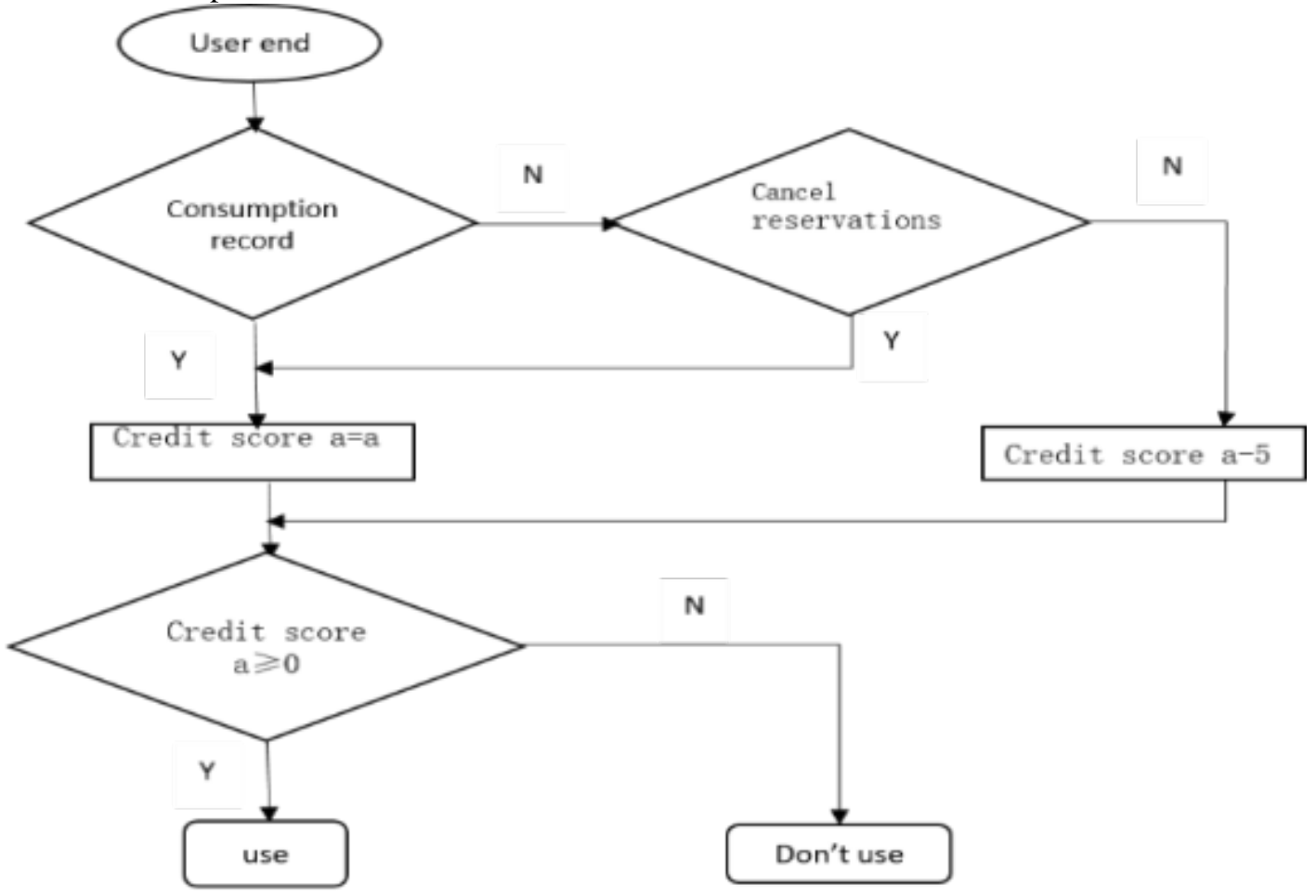

Figure 7. credibility testing flow chart 


\section{RESULt}

This paper bases on traffic requirements of university students, builds an intelligent traffic system with features of easy implementation and having 'custom-style'. We can conclude it as a 'Multiple-Ring' system with one core and five parts. (Figure 8)

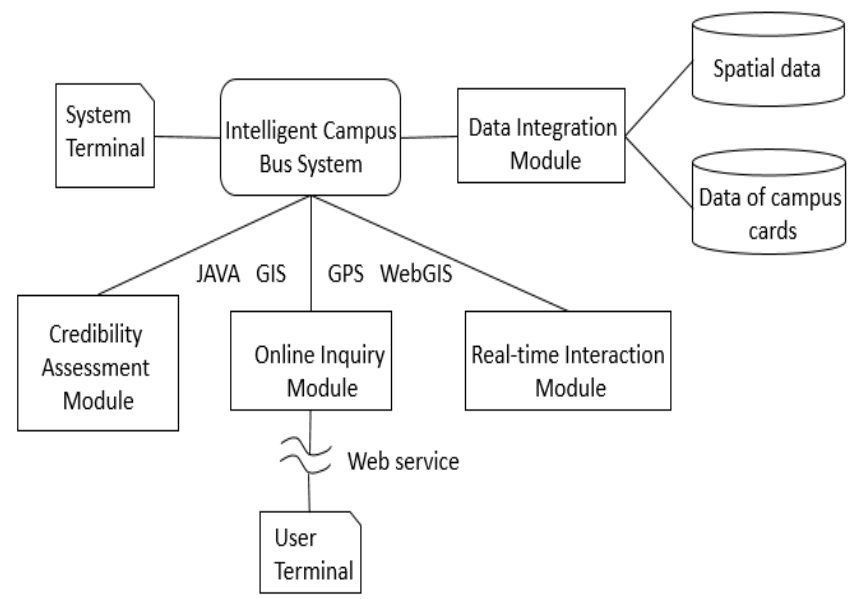

Figure 8. System diagram

\section{REFERENCES}

[1] M Xu , XD Pan et al. Intelligent Traffic System Design Research of Urban Complex Underground Garage. Applied Mechanics \& Materials, 2015,743:715-723

[2] 2. Maciaszek, L A. Requirements Analysis and System Design. Beijing: China Machine Press.2009.

[3] 3. XH Qian et al. Intelligent transportation. Beijing: Tsinghua University Press. 2015.57-58.

[4] 4. N An, and Z Zhang. "Real-Time Query Client on Mobile Platform in Intelligent Transportation System”. Computer Systems \& Applications. 2014,23(1):176-180.

[5] 5. GY Cheng and JC Wang. A Review of Data Integration. Computer Science. 2004,05:48 51.

[6] 6. $X$ Zhang. The Design and Implementation of Qingdao Integrated Query Convenient Information Service Platform Based on Android Platform. Shandong: Shandong University.2015.

[7] 7. QS Liu. The design of metaheuristic algorithm about School Bus Routing Problem and the Integration of GIS. Henan: Henan University.2013.

[8] 8. J Fu et al. "The application of GIS in the designing of tourism routes". Journal of Shanghai Normal University: Natural Sciences. 2006,35(3):92-97. 